\title{
Renégats marseillais (1591-1595)
}

\author{
GABRIEL AUDISIO
}

$\mathrm{L}$ attention des historiens et d'un public plus large de lecteurs a été récemment attirée par $B$. et $L$. Bennassar sur les chrétiens qui étaient passés à l'islam aux seizième et dix-septième siècles. ${ }^{1}$ Comblant ainsi une lacune évidente de l'historiographie occidentale - que la place récemment prise par le monde musulman dans nos préoccupations permet sans doute d'expliquer - cette belle étude fait le point sur la question, à partir de 1550 cas individuels tirés des archives de l'Inquisition, espagnole principalement. La présente réflexion ne prétend en aucun cas se substituer à elle. Bien mieux, elle suppose sa lecture et, une bonne fois pour toutes, y renvoie le lecteur désireux de poursuivre sa réflexion dans cette voie et auquel l'ouvrage de B. et $L$. Bennassar aurait échappé.

Parmis les 171 Français, soit $11 \%$ de l'effectif recensé, les auteurs dénombrent 60 Provençaux, dont 29 Marseillais. ${ }^{2}$ Il n'est pas étonnant que la cire phocéenne ait fourni le lot principal des renégats de Provence, soit la moitié. Le port méditerranéen, presque entièrement tourné vers la Barbarie, faisait grand commerce avec les rives du Maghreb. Il ne fait aucun doute qu'une enquête minutieuse sur ce site augmenterait considérablement le nombre de Marseillais détenus ou résidant en terre d'islam, comme le suggère un rapide sondage présenté ci-dessous. En tout état de cause c'est en complément apport d'un modeste caillou à l'édifice - que se situent ces quelques pages.

A la faveur d'une invitation pour donner une conférence à l'Université de Madison-Wisconsin (Etats-Unis) en 1986, j'eus la possibilité de discuter avec John Tedeschi. Celui-ci, fort obligeamment, m'indiqua alors - avant d'en révéler plus tard publiquement l'existence - un registre de l'inquisition romaine égaré à la Bibliothèque Nationale de Paris, témoin de l'aventure parisienne que connurent les archives romaines au début du dix-neuvième siècle. C'est là que je découvris les procès intentés par le tribunal ecclésiastique à cinq renégats français entre 1592 et 1595: un provençal, sans autre précision, et quatre marseillais. Par ailleurs, un registre notarié de Marseille contient, perdus dans un ensemble d'actes de l'officialité de ce diocèse, la 
réconciliation effectuée en 1591 pour trois Marseillais renégats. Ces huit hommes ne figurent évidemment pas dans la liste publiée par B. et L. Bennassar, et pour cause. ${ }^{3}$

Apporter, grâce à ces nouveaux témoignages, quelque complément d'information à la masse déjà rassemblée et publiée n'est pas inutile. Cela permet, par une sorte d'effet de zoom sur un site, de préciser nos connaissances sur les renégats marseillais et de vérifier à cette occasion que, outre les archives de l'Inquisition espagnole, il convient à qui voudrait étudier les renégats originaires d'une même localité, de ne pas négliger, suivant le cas, celles de l'Inquisiton romaine ou des officialités et, autant que possible, les registres notariés. Pourtant cela ne justifierait guère une nouvelle publication car, comme nous le verrons, les déclarations de nos renégats ne tranchent guère par rapport à celles des autres Marseillais qui, dans des situations semblables, ont comparu devant le tribunal de l'Inquisition espagnole. L'intérêt principal est ailleurs. Le présent article voudrait attirer l'attention sur deux autres aspects. Le premier est une approche de la conception religieuse qu'avaient les hommes de ce temps: les clercs qui interrogent et les renégats qui répondent partagent-ils la même vision? Le second est l'embarras dans lequel se trouvent certaines autorités ecclésiastiques auxquelles échoient ces cas. Elles ne savent comment procéder. Plusieurs lettres échangées entre des évêques italiens et le Saint Office, conservées dans le même registre, permettent d'assister à la gestation d'une procédure particulière adaptée aux renégats. Par ailleurs, une nette différence apparaît dans le traitement de l'apostasie entre l'Italie et la France. Mais, dans un premier temps, ouvrons le dossier et faisons connaissance avec ces hommes.

\section{Contraints à l'aventure}

Puisque le nombre en est réduit, examinons les cas et prenons-les dans l'ordre où ils ont été présentés au tribunal. Nous pouvons ainsi suivre leurs péripéties, du moins dans la version qu'ils ont présentée au tribunal. ${ }^{4}$

Jacques Gérard comparaît le 2 septembre 1592 devant le tribunal établi dans les prisons du palais archiépiscopal de Naples. L'accusé déclare avoir 17 ans, être fils de Pierre et de Géminette de Cabarne, et marin de profession. Son incarcération a commencé en mai à Cosenza. Mais comme il déclara qu'il avait renié la foi chrétienne en Barbarie et qu'il voulait être réconcilié avec l'Eglise, il fut envoyé à Naples. L'inquisiteur l'invita à raconter comment il avait renié la foi et à dire tout ce qu'il avait sur la conscience, pour le seul salut de son âme. Marin, il se trouvait deux ans auparavant - il avait alors 15 
ans - sur un galion appartenant à un patron provençal, partant de Ligurie chargé de sel et revenant en Provence. Au large, le bateau fut pris par la galiote d'un certain Mamet et tous se retrouvèrent à Bizerte un mois plus tard. Lui-même fut vendu au comité de la galiote nommé Pirroloisi, un catalan renégat. Celui-ci le harcelait pour qu'il se fit turc. Le prisonnier résista aux mauvais traitements et aux pressions pendant un mois. Mais comme son maître lui promit de le libérer et de le mettre à la tête de sa maison, il céda.

Il fut alors "retagliato", c'est-à-dire circoncis: "Quand je fus circoncis je fus lié au pied du mât de la galiote, car je ne voulais pas être circoncis. Me voyant circoncis, je levai la main, huit jour après, disant les paroles habituelles à ceux qui renient la foi chrétienne c'est-à-dire: Lei le ella llà, Maumet del Sole lla. Dès lors il a fréquenté les bains "comme il est usuel chez les Turcs" car les Turcs prétendent qu'en se lavant ils effacent leurs péchés, a suivi la loi des Turcs, s'est vêtu en turc, a mangé de la viande le vendredi et le samedi, est allé à la mosquée cinq ou six fois avec son patron. Il ne savait s'il faisait bien ou mal, car il n'était qu'un enfant. Mais dès lors il ne songeait qu'à s'enfuir.

A la faveur d'une corvée d'eau, au cap des Alici, vers Cotrone en Calabre, à la Saint Jean-Baptiste dernière, soit le 24 juin, il s'enfuit et se cacha dans un bois, puis se réfugia dans un monastère. Le lendemain le capitaine vint le chercher, le conduisit chez lui et lui fit réciter Pater Noster, Ave Maria, Credo et Salve Regina. Puis il fut emmené à Cosenza pour être réconcilié, où il resta dix mois, puis fut envoyé à Naples, où il se trouve emprisonné depuis le mois de mai précédent. Il fut entendu une fois, mais les juges ayant appris qu'il était circoncis, il ne fut plus convoqué. Pendant ces dix mois de détention il a observé la loi turque, mais seulement extérieurement. Sa volonté a toujours été d'être chrétien. Ainsi il récitait secrètement les sept psaumes, qu'il lisait dans un petit office qu'il avait, ainsi que Pater Noster et Ave Maria et faisait le signe de la croix. Il est baptisé et il se souvient qu'à dix ans il fut confirmé par l'évèque de Saint-Paul en Provence ${ }^{5}$ et, avant sa prise par les Turcs, il se confessait une fois par an pendant le carême, mais il ne communiait pas car il n'avait pas l'âge.

Tel est le récit de Jacques Gérard, qui signa sa déposition mais dont nous ignorons par ailleurs le sort, à l'issue du procès. Gageons que, sans trop de difficultés, il fut admis à abjurer.

Jean Fabre, lui, est marseillais et n'est resté que six mois aux mains des Turcs. Voyant qu'il ne pourrait se libérer que par la fuite, il déclara qu'il voulait se faire turc. Moyennant cette promesse il fut tiré de la chaîne, mais il n'eut jamais cette intention. Ces jours passés, arriva en Sardaigne un bateau 
de Sa Sainteté chargé de blé et venant de Sicile qui fut pris par des galères turques. Sur l'une d'elles se trouvait Jean Fabre. Or les Turcs manquaient d'hommes pour conduire le navire en Barbarie. Ils proposèrent donc aux quatre esclaves français qu'ils avaient pris la veille de mener le bateau en Barbarie moyennant leur liberté, la restitution de leurs biens et le versement d'une bonne somme d'argent. Les Français acceptèrent. Toutefois six Turcs et notre Jean Fabre leur furent attribués comme compagnons et gardiens. Tout en naviguant, "et étant presque proches de la Barbarie", d'accord avec les Français, ils rebroussèrent chemin et vinrent vers Civitavecchia avec le consentement du Marseillais. Ainsi ce dernier se trouva alors enchaîné avec les Turcs pour avoir seulement renié en paroles la foi, mais jamais en fait; d'ailleurs il n'est pas circoncis. Ordre est donné de le transférer à Rome, sur sa demande, pour y être examiné.

La séance reprend le mardi 4 mai 1593 dans le palais du Saint-Office, à Rome. Nous apprenons que Jean Fabre est fils de François et qu'il a 26 ans environ. Il fait alors le récit de sa capture. Parti de Marseille sur une barquette avec six compagnons pour aller charger du vin, il fut fait prisonnier à l'île d'Hyères, lui et ses compagnons, par le raïs Amet Bais qui les mena en Barbarie. Ses compagnons furent vendus. Mais lui, comme il connaissait l'art de la navigation, il fut gardé par Amet Bais "lequel voulut me garder pour son usage". Et dès lors il le pressait de se faire turc, lui promettant la liberté et autres choses. Devant sa résistance, le raïs commença à le menacer de le jeter dans une tour, à lui faire divers maux et déplaisirs. Or Jean Fabre, ayant femme et enfants, désirait trouver le moyen de s'en retourner en Chrétienté. Pensant que s'il ne lui faisait cette promesse il courait le risque de ne jamais recouvrer la liberté et donc de ne jamais pouvoir s'enfuir, il se résolut - "bien contre son gré" - à lui promettre de vouloir se faire turc. Amet Bais le fit aussitôt libérer de ses chaînes et lui donne une épée pour combattre en cas de besoin. Et ils partirent ensemble de Sardaigne avec quatre galiotes.

Doublant le cap "Taurario", ils arrêtèrent un vaisseau français. Mais comme il avait la "patente" du Grand Turc, ils le laissèrent aller, se contentant de faire prisonniers quelques Génois qui se trouvaient à bord. Plus loin ils rencontrèrent un esquif avec quatre hommes à bord: deux se sauvèrent à terre; les deux autres, moins lestes; furent faits prisonniers. L'un d'eux se nommait Jean Morin, également marseillais. Parvenus dans un port de Sardaigne nommé S. Agalgliano, ils trouvèrent un navire chargé de blé mais abandonné par les matelots qui avaient vu les galiotes. Amet Bais et les Turcs se réjouirent de cette prise qui leur assurerait la fortune à condition de ramener l'embarcation en Barbarie, où le blé se vendait cher. "Mais les Turcs ne sont pas habiles 
à conduire les bateaux." Ils demandèrent donc à Jean Fabre s'il se sentait capable de conduire la cargaison en Barbarie. Celui-ci, saisissant l'occasion espérée, l'attesta, avançant sa compétence à la boussole comme aux cartes maritimes. Amet Bais lui confia alors le bateau chargé de blé et lui promit la liberté s'il le conduisait à bon port.

Aussitôt installé au timon et le large gagné, le marin marseillais dit aux quatre autres chrétiens, que le raïs lui avait donnés pour le voyage, qu'ils tenaient l'occasion de s'en retourner tous ensemble en Chrétienté. S'ils avaient besoin d'armes, il pourrait leur en fournir puisqu'il avait la clef de la réserve. Ainsi fut-il décidé. Se trouvaient à bord cinq chrétiens et six soldats turcs. Pour pouvoir s'en venir en Chrétienté sans éveiller les soupçons, il alla en haute mer, voulant aborder à Civitavecchia. Au bout de six jours les turcs commencèrent à avoir des soupçons, disant qu'ils ne croyaient pas qu'il fallût autant de temps pour gagner les côtes de Barbarie. Mais il leur répondit que le vent était faible. Surtout il leur indiqua que se trouvait à bord un grand et fameux vin, qu'il leur donna à boire. Aussi les soldats se tinrent-ils joyeux, à moitié saouls. Ils arrivèrent ainsi en vue de Civitavecchia. Ce que voyant, les Turcs doutèrent de lui, affirmant que ce n'était pas la Barbarie mais terre des chrétiens. Fabre leur répondit qu'il avait été trompé par la boussole. Comme ils n'y entendaient rien, ils le supplièrent de les conduire en France ou de les laisser sur quelque île. Le Marseillais leur répondit qu'il valait mieux pour eux d'être conduits à Civitavecchia, qui était terre du pape, car le pape était prince saint et bénigne et que, lui amenant un bateau chargé de blé, ils ne couraient aucun danger, bien mieux ils recevraient 50 écus chacun et une "patente" de liberté. Ainsi ils s'apaisèrent et tous entrèrent au port, où les Turcs furent faits prisonniers, Jean Fabre compris.

Interrogé par l'inquisiteur, Fabre répond que durant son séjour avec Amet Bais il n'a jamais fait aucun acte de turc, même pas en pensée. Au contraire, il s'est toujours recommandé à Dieu pour qu'il l'aidât à se soustraire de leurs mains. Il n'a jamais cru ni voulu croire en leur secte.

Le 6 mai, Jean Fabre était absous et réintégrait la Sainte Eglise moyennant une pénitence bien légère: réciter trois chapelets, se confesser puis visiter les sept églises de Rome, et séance tenante, il était libéré.

Léon de Olivier, marseillais, environ 25 ans, comparaissait le 23 juillet 1593 devant l'inquisiteur dans le palais du Saint-Office à Rome. Nous pouvons résumer ainsi les informations qu'il a livrées au cours de son long interrogatoire. Il avait quitté sa ville natale douze ans auparavant - il avait donc alors 13 ans - sur un gros bateau nommé Sainte Anne dont le patron était Nicolas Chiche, de Marseille, pour livrer des draps et autres marchan- 
dises à Alger. Mais, parvenus à une trentaine de miles de la Barbarie, ils rencontrèrent la galère d'un certain raïs nommé Cinq Dents. Celui-ci arraisonna le bateau et s'empara des balles de draps de soie et de Léon, "parce que j'étais jeune garçon (giovanotto)", mais il laissa les autre hommes. Cinq Dents le consuisit à Bizerte où il le vendit à un janissaire nommé Amurat. Dès qu'il l'eut acheté ce dernier commença à lui dire qu'il devait se faire turc. Et comme il ne voulait pas, il lui donnait la bastonnade quotidienne. Un jour, après un mois de ce traitement et pour éviter les coups de bâton, il lui dit qu'il voulait se faire turc. Aussitôt trois janissaires vinrent le circoncire; dès lors il n'eut plus de bastonnade. Et quand il se fit turc il "leva le doigt et dit telles paroles qui se disent habituellement, à savoir, Ley lala Mahumet resula, qui signifient qu'ils ont gagné une âme". Il reçut alors le nom de Mami et s'habilla à la turque. ${ }^{6}$

Toutefois il est toujours resté bon chrétien: il évitait de manger de la viande les vendredis et samedis et il n'a jamais voulu apprendre leur loi ni leur "messe". Quand il sortait, il disait le Pater Noster et l'Ave Maria. Il fut aussitôt invité par le tribunal à reciter ces prières. Ce qu'il fit, avec toutefois des omissions et des erreurs. Au juge qui le lui fit remarquer il se contenta de répondre: "Mon père me les a enseignées de cette manière". De plus il se révèle incapable de dire d'autres prières, déclarant: "Le credo et le Salve Regina, je ne les sais pas". Pendant tout le temps qu'il est resté en "Turquie" il n'est jamais allé a la mosquée. Il est bien passé devant, mais il n'y est jamais entré. Son patron, qui était soldat, avait l'habitude d'aller à la mosquée une fois la semaine, le jeudi, mais, lui, restait à la maison. Quand arrivait Noël, il allait à Tunis à l'église des chrétiens. Un fois même le Bey de Tunis voulut le faire brûler parce qu'il avait entendu dire qu'il était allé dans l'église des chrétiens, mais des janissaires de ses amis lui dirent que c'était pour se promener et pour aller à la taverne avec des chrétiens, vu qu'il était de nation française.

Après neuf ans passés au service du même patron, comme ce dernier avait préparé le papier de sa libération, à sa mort, il se trouva libre. Il devint alors soldat à Tunis sous les ordres du Bey ou roi de cette cité, et cela pour trois ans. Il resta célibataire et, durant sa vie militaire, il vivait avec cinq autres soldats de nation turque. Mais chacun mangeait sa propre nourriture, car le roi donnait à chacun sa paye de quatre écus par mois. Il se trouva un jour à bord d'un frégate appartenant à Ade raïs venant faire la course. Ils étaient en tout 25 hommes "di bona voglia" (volontaires) et devaient avoir part au butin. La frégate devait recevoir six parts, dont une pour le raïs. Parvenus près de ces plages, ils furent découverts par vingt-deux galères qui les prirent en 
chasse. Ne pouvant se sauver par mer, "vu qu'ils étaient plutôt esclaves de terre que des galères," ils allèrent avec la frégate à terre et là ils furent pris par les gens du seigneur Don Virginio Orsino. Celui-ci fit conduire à Rome Léon de Olivier ainsi que neuf autres de ses compagnons qui se trouvaient sur la frégate. Voilà comment il se trouve dans ce palais du Saint-Office.

$\mathrm{Au}$ terme de ce premier interrogatoire, le tribunal ne fut pas convaincu. Comme il est précisé dans le procès-verbal, “il n'est pas vraisemblable que son patron Amurat, qui le fit circoncire, lui ait permis de vivre à la façon des chrétiens, car les Turcs n'ont pas coutume de le permettre aux circoncis". C'est pourquoi le juge le laissa méditer quarante jours en prison avant de le convoquer à un nouvel interrogatoire, le 1er septembre 1593, au cours duquel il maintint ses premières dépositions. Nouvelle séance le 25 septembre. Il est clair que le tribunal le soupçonne de s'être vraiment converti à l'islam et d'être venu en course contre les chrétiens. L'accusé proteste: s'il est venu vers l'Italie, ce n'est pas pour la course et s'il est resté tant d'années chez les Turcs c'est parce qu'il n'a pas eu l'occasion de revenir avant. Mais son intention était de retourner à la foi chrétienne. Venu une fois en Corse, il essaya de fuir mais ses compagnons turcs le firent remonter sur la frégate.

L'accusé reste suspect aux yeux du tribunal qui l'invite à dire la vérité avant de lui appliquer la question. Il s'inquiète: "Je n'ai rien pour vivre, je suis pauvre... Ayez pitié de moi. Si vous m'estropiez, je ne serai plus bon à rien”. Il tient à préciser que lorsqu'ils abordèrent, il alla spontanément au devant des cavaliers qui les cherchaient, leur disant qu'il était français et qu'il voulait rester bon chrétien. Ils lui demandèrent où étaient les Turcs et lui dirent de les appeler. Ils les appela et ils arrivèrent six, avec lesquels il fut amené, accompagné du châtelain, sur cette terre du seigneur Don Virginio Orsino. Ces précisions n'eurent pas plus d'effet sur le juge que les précédentes déclarations. Pendant qu'on lui enlève ses vêtements, Léon de Olivier gémit: "Je suis pauvre. De grâce ne m'estropiez pas". Pressé encore de dire quel genre de vie et quelle croyance il avait chez les Turcs, il répète qu'il a toujours cru en Dieux, en la benoite Vierge Marie qui doit l'aider, qu'il n'a jamais pensé que les Turcs puissent se sauver, eux qui ne croient ni à la madone ni aux saints.

Il est alors “élevé”, c'est-à-dire sans doute soumis à l'estrapade. Sous la torture, il résiste. Il laisse échapper des plaintes tout en maintenant ses déclarations: “O Dieu, je vous demande pardon... miséricorde... On me casse les bras... O Jésus bénit, que l'on me croie ici; je vous demande pardon, ayez pitié de moi... Je vous ai dit ma façon de vivre chez les Turcs, voilà la vérité. Ayez miséricorde de moi”. Puis il se tait. Puis: "Dieu du paradis, ayez pitié”. 
Puis il se lamente disant: “Dieu, ô Dieu!". Ensuite, il se tait. Pressé de dire s'il n'a pas abandonné le christianisme et adhéré à la secte mahométane, il répond: "J'ai toujours cru en Dieu et en la Vierge Marie et Dieu le sait. Je vous demande miséricorde de moi qui suis mort". Aux autres questions, il se contente de répéter qu'il ne sait pas, qu'il a dit la vérité. Ordre est finalement donné de le descendre, le détacher, le revêtir et le reconduire en sa prison. La séance de torture a duré un quart d'heure. La suite et l'issue nous sont inconnues.

Vital Redon, autre Marseillais, fils de François, menuisier, 37 ans environ. Huit ans avant sa comparution, il était parti de Marseille pour s'installer à Malte, où il avait une femme et un fils qui, au moment de l'interrogatoire, devait avoir onze ans. Il se mit d'accord avec un autre Provençal, Pierre de Nille, qui possédait un bateau et allèrent charger du blé à Agrigente pour le porter à Malte. Sur le chemin du retour, le bateau fut pris par trois galiotes turques et, quant à lui, il fut conduit à Constantinople, où il fut présenté à Abraïm Bascia, gendre du grand Turc. Entravé, avec d'autres esclaves, il devait transporter du bois. Et il resta ainsi esclave huit ans, durant lesquels il tenta sept fois de s'enfuir: trois fois par terre et quatre par mer. La quatrième fois ils lui coupèrent l'oreille et pendant un an il resta au collet avec les menottes; et chaque évasion lui valut des bastonnades. C'est ainsi qu'il renia, pour avoir plus de facilité à fuir, comme le montra sa dernière tentative.

Chez le même patron, se trouvait aussi un renégat hongrois, Jean de Maggiara, avec lequel il prépara son évasion: ce dernier par terre et lui par mer. Voyant qu'il voulait monter à bord, les marins turcs lui demandèrent s'il appartenait à quelque patron. Il répondit qu'il était turc mais qu'il voulait partir à cause de dettes et qu'il avait peur de la justice. Alors ils le cachèrent sous sept couvertures. Embarqué, avec deux galiotes qui allaient à Alger, il s'arrêta au cap de Marso habité par les Grecs, où arriva un bateau de Candie (Crète) chargé de vin qui le conduisit à Corfou. De là, sur une barque, il parvint à Otrante. Puis, allant de terre en terre, il longea la côte espérant arriver à Rome. Il aborda ainsi à S. Mauro où, demandant l'aumone, il fut pris par les gens du comte qui l'emprisonnèrent sous pretexte qu'il était un espion des Turcs alors qu'il demandait, lui, qu'on le conduisit devant le comte, car il voulait lui dire la vérité. Mais ils ne consentirent jamais à l'y conduire. Ainsi il se retrouva dans cette ville de Santa Severina, et dans ce palais archiépiscopal, où se tient son procès ce 11 décembre 1593.

Il a renié la foi chrétienne de bouche seulement et non de coeur, bien entendu, et seulement à cause des bastonnades qu'on lui donnait ainsi que pour avoir une plus grande commodité de fuir. Il en parla avec un frère de 
l'ordre de Saint François, Horace Piacentino, lequel lui dit qu'il ne saurait lui conseiller ni oui ni non; que, s'il le faisait pour fuir, il aille vers le pape, car il l'absoudrait; mais que, s'il le faisait pour rester en Turquie, il serait damné en enfer. Et il lui répondit qu'il le faisait de bouche non de coeur et pour s'enfuir. Il fut alors circoncis le 7 mai 1593, et parvint à fuir le 29 juin. Le récit parut suffisamment convaincant au tribunal pour qu'il soit relâché aussitôt, sans aucune pénitence. Non seulement Vital Redon n'était pas un espion mais il apparut comme une pure et malheureuse victime des Barbaresques.

Augustin Borré, également marseillais, 20 ans, est interrogé dans le palais épiscopal de Mileto en Calabre, le 19 janvier 1595. Pris par les Turcs cinq ans auparavant, il fut forcé de renier la foi chrétienne et circoncis à la mode des Turcs quelques jours après sa capture. Il renia à cause des mauvais traitements qu'il subissait et des bastonnades qu'il recevait; et quand il fut circoncis, ils étaient quatre à lui tenir les mains et les pieds pour le circoncire de force. Il vécut ensuite cinq ou six ans en terre turque mais il n'a jamais cru que la secte des Turcs soit bonne ni propre à faire le salut des gens. Il n'a jamais abandonné la foi du Christ. Au contraire il a toujours eu la volonté d'y retourner. Le soir il disait secrètement le Pater Noster, l'Ave Maria et le Credo et il faisait le signe de la croix. Une fois un religieux lui donna un chapelet qu'il garda toujours avec lui pendant environ six mois. Il s'est finalement enfui de chez les Turcs pour revenir à la foi chrétienne afin de sauver son âme "parce que comment celui qui n'a pas la foi du Christ veut-il sauver son âme!"

Ce récit, malgré quelques points douteux, a convaincu l'évêque de Mileto qui fait abjurer l'islam à Augustin Borré, l'absout et le réintègre dans le giron de notre sainte mère l'Eglise sans délai ni pénitence.

Restent les trois derniers, retrouvés dans un registre tenus par un notaire de Marseille, greffier de l'officialité de ce diocèse, Renaud Cappus, Pierre Fanti et Honoré Dalhot se sont présentés spontanément devant le tribunal. On peut se demander d'ailleurs si, de retour dans leur cité d'origine, ils pouvaient faire autrement. Nous sommes moins bien renseignés sur eux car, à la différence des renégats retrouvés sur les côtes italiennes, les documents marseillais sont plus brefs. Nous savons cependant que tous trois furent pris en mer par les Turcs sur un bateau marseillais alors qu'ils avaient 14, 12 et 13 ans et conduits à Alger où, de force, ils furent contraints de renier la foi et ils furent circoncis. Toutefois, comme les précédents et selon leurs dires, le reniement ne fut que de bouche et non de coeur. Renaud Cappus, examiné le 10 juillet 1591 et alors âgé de 18 ans, ainsi que Pierre Fanti, comparaissant deux jours plus tard et âgé de 26 , durent leur libération à la prise des trirèmes 
turques sur lesquelles ils étaient par des Génois qui les tinrent prisonniers plusieurs années. Mais quelques jours auparavant les galères génoises vinrent mouiller dans le port de Marseille et, sur place, leurs parents obtinrent leur libération, sans doute moyennant finance, ce qui n'est pas précisé. Le dernier, Honoré Dalhot, examiné le 8 septembre 1591 et âgé de 14 ans, avait été pris par les "chiens Turcs infidèles" dix-huit mois plus tôt seulement, sur un navire marseillais qui naviguait vers l'Espagne. Le mois précédant sa comparution, ayant quitté Alger sur une trirème turque pour la Corse, sous la conduite de son patron Morat raïs, il parvint, à s'enfuir secrètement et se cacha un jour et une nuit dans les bois de l'île jusqu'à ce que les Turcs aient pris le large. Il réussit ensuite à gagner une trirème du grand duc de Toscane, de laquelle, début septembre, il débarqua à Marseille. Tous trois furent réconciliés sans difficulté; les deux premiers se virent imposer comme pénitence de communier à la fête de l'Assomption, soit le 15 août suivant, le dernier ne reçut aucune pénitence ${ }^{7}$.

\section{Une religion concrète}

Ces huit témoignages, on le voit, présentent à la fois suffisamment de similitudes pour offrir une sorte de schéma type du renégat et suffisamment de différences pour permettre de saisir le destin personnel de chacun. Par les détails de leur "récit de vie" raconté au tribunal, ils nous permettent de nous faire une idée des circonstances du passage à l'islam et de la condition de vie des renégats, compte tenu toutefois du stéréotype qui les présente toujours comme des victimes de la barbarie "turque," restées fidèles à la religion chrétienne malgré souffrances et tourments. Ces témoignages nous fournissent aussi des indications sur les raisons qui poussent les corsaires musulmans à s'emparer de certains jeunes chrétiens et à les convertir. Ils nous informent aussi sur quantité de questions matérielles ou concrètes: types de bateaux, marchandises et sites du commerce méditerranéen. Nous y voyons la place particulière que tient la France dans ces relations avec la Porte, grâce aux "patentes," c'est-à-dire les capitulations.

Quoique différents dans le détail, ces récits présentent suffisamment de points communs pour permettre de dresser une sorte de portrait robot du renégat marseillais. Tout d'abord, c'est un marin. Un seul des huit, Vital Redon, ne l'est peut-être pas, encore que l'on comprenne mal, dans ce cas, comment il se trouvait à bord d'un navire faisant le commerce du blé. Que ces hommes soient des “mariniers" n'a pas de quoi surprendre puisqu'ils habitent Marseille. Leur âge est une autre donnée remarquable. Lors de leur 
interrogatoire, la moyenne d'âge est de 23 ans, moins élevée que celle des 29 autres Marseillais retrouvés par L. et B. Bennassar qui est de 27 ans. Mais plus intéressant est leur âge lors de leur capture: le plus jeune avait alors 12 ans et le plus âgé 29, la moyenne étant de 17. Pour 22 des 29 Marseillais retrouvés par $\mathrm{L}$. et $\mathrm{B}$. Bennassar, nous connaissons l'âge à leur capture: le plus jeune avait 8 ans et le plus vieux 25, la moyenne se situant à 16 ans et demi. Malgré la faiblesse de l'échantillon marseillais qui se réduit à huit personnes, la convergence est frappante. Il s'agit donc bien toujours de jeunes gens et même assez souvent d'enfants, pourtant déjà entrés dans la vie professionnelle, ce qui n'avait alors rien d'exceptionnel. Voilà qui atteste le goût des Barbaresques pour les jeunes mâles. D'ailleurs une remarque de Léon de Olivier le confirme: lorsqu'il fut pris, il avait 13 ans; Cinq Dents s'empara de lui "parce qu'il était giovanotto" et délaissa les autres chrétiens du bateau.

Le récit des aventures vécues par ces renégats présente également des similitudes remarquables. La capture se fait toujours en mer, au cours d'un trajet à but commercial. Conservé par le capitaine du bateau barbaresque ou vendu à un nouveau patron, le jeune homme saisi se retrouve dans un grand port de la Méditerranée: Bizerte, Constantinople, ou quelque part en "Barbarie", mais surtout à Alger. Là, les mauvais traitements commencent, notamment la bastonnade, pour l'obliger à se faire "turc". La resistance à l'apostasie est plus ou moins longue, mais toujours réelle ou, en tout cas, affirmée. Finalement, le malheureux cède pour deux raisons principales avouées: faire cesser les coups et obtenir la liberté, propice à l'évasion. La cérémonie a lieu: le converti prononce les paroles prévues en levant le doigt vers le ciel: "La ilaha illa Allah Mohammed rezul Allah" et dès lors il reçoit un nom musulman et s'habille à la turque. ${ }^{8}$ Mais ce n'est là qu'apparence. Dans son coeur, il est resté chrétien. Il va le moins possible à la mosquée, ou même jamais; évite de manger de la viande les vendredis et samedis; récite en secret les prières du Pater Noster et de l'Ave Maria. Bref, à aucun moment il n'a cru pouvoir faire son salut dans l'islam et, rêvant de revenir en Chrétienté, il guette la première possibilité d'évasion. Comme c'est un marin et qu'il vit dans un port, c'est par la voie maritime qu'il étudie sa fuite. Finalement, grâce à la ruse et à la chance, il parvient en terre chrétienne quelque part sur les côtes de l'Italie du Sud ou sur quelque île, à moins qu'il ne soit fait prisonnier par une flotte chrétienne, comme le furent deux d'entre eux par les Génois. Dès qu'il rencontre les représentants de l'autorité locale, il leur déclare vouloir réintegrer la communauté chrétienne. Emprisonné, il est conduit au tribunal 
ecclésiastique qui examine son cas. Tel est le schéma général de l'aventure de nos renégats.

Ces conclusions rejoignent celles de L. Rostagno ou de L. et B. Bennassar. Il est donc inutile d'insister ici, ce simple rappel aura suffi. Retenons seulement un aspect particulier: l'idée que les renégats, connaissant à la fois christianisme et islam pour les avoir pratiqués l'un et l'autre, se faisaient de la religion en général et du christianisme en particulier. Je ne prétendrais certes pas que cet aspect n'a pas déjà été étudié ailleurs. De nombreux auteurs l'ont abordé, dans une perspective particulière, d'ailleurs liée aux sources utilisées, pincipalement littéraires. Pour leur part et dans le champ historique, L. Rostagno comme L. et B. Bennassar n'ont également pas manqué de le faire. ${ }^{9}$ Ces auteurs ont bien fait ressortir l'image de l'islam que se faisaient les renégats - ce qui d'ailleurs laisse entière la question de l'éventuelle déformation volontaire ou inconsciente de la part de ces transfuges. Toutefois il me semble qu'un autre regard interrogateur peut se poser sur ces témoignages pour y rechercher dans un premier temps le type de christianisme que reflètent tant les clercs que les renégats et, dans un second temps, les oppositions certes, mais aussi les convergences, entre christianisme et islam qui transparaissent au travers de ces expériences. ${ }^{10}$

Le caractère le plus frappant qui ressort à la lecture de ces interrogatoires est que, pour tous, aussi bien les clercs qui interrogent que les laïcs examinés, la religion est profondément incarnée et se manifeste concrètement dans la vie quotidienne. Bien représentatifs de leurs contemporains sur ce point, ils participent de cette vision globale selon laquelle la croyance "informe" tout l'être et son expérience vécue. La religion est partie intégrante d'une civilisation, d'une culture. A cet égard le vocabulaire est significatif. Dans la bouche des renégats, comme sous la plume des clercs, "turc", "mahométan" et "barbaresque" sont synonymes. Et, pour eux, "chrétienté" est un concept géographique tout autant que religieux. Cette vision schématique ne fait place à aucune nuance, aucune exception, aucune minorité. Pourtant, on le sait, la réalité était loin de correspondre à une partition en deux blocs aussi massifs et la frontière était bien plus floue et fluide qu'il n'y paraît. Qu'importe! Telle est la conception du temps. Par le mode de vie apparent, dès lors, il est possible et même aisé à un familier, voire à un passant, d'induire l'appartenance religieuse d'un individu. C'est dans ces éléments les plus concrets, les plus matériels presque, que se marque la différence et même l'opposition entre les deux religions. On mange, on s'habille, on vit "à la turque" ou en chrétien. Les deux voies sont exclusives; impossible, à les entendre, d'établir je ne sais quel compromis commode entre Jésus et Mahomet. La plupart de leurs 
contemporains ne se posent guère la question mais les renégats, eux, ont dû choisir, et au moins deux fois.

D'après leurs déclarations, devenir musulman c'est être circoncis, prononcer la phrase de profession de foi mahométane, changer de nom; et pratiquer l'islam c'est s'habiller à la turque, manger de la viande le vendredi, aller aux bains et parfois à la mosquée. Nous ne savons pas si les clercs partageaient une telle vision. Je doute qu'elle ait été aussi "matérielle", au risque d'ailleurs d'un contre-sens, établi dans l'affectation indue de données chrétiennes à l'islam. Nous pouvons le supposer, vu les divergences que nous constatons entre la vision du christianisme que présentent les renégats et celle des clercs.

Ecoutons les renégats lorsque, invités par le tribunal à raconter comment ils ont été pris par les Barbaresques et comment ils ont vécu chez eux, ils parlent sinon sans contrôle du moins sans contrainte. L'expérience que j'ai des procès inquisitoriaux m'incite à penser que, sur le plan méthodologique, il convient, de distinguer soigneusement les informations livrées par un prévenu qui parle de son propre mouvement - sponta sua - de celles qui viennent en réponse à une question précise du juge. Non seulement la fiabilité du contenu mais aussi la signification même de l'expression en dépendent. $\mathrm{Au}$ moment qui nous intérésse et qui se situe généralement au début de l'interrogatoire - la précision est d'importance car le renégat n'a encore subi aucune pression et peut légitimement croire que la tribunal est bien disposé à son égard - se place son récit de vie. Dans cette partie du procès il parle spontanément de la manière dont, malgré son reniement et un environnement particulièrement peu favorable, il est resté chrétien de coeur. Tout pousse donc le renégat à montrer l'intériorisation de sa religion. Or qu'offre-t-il à l'inquisiteur qui l'interroge? Un religion tout extérieure. D'après ces témoignages, être chrétien c'est, implicitement et par opposition à l'islam, s'habiller à l'occidentale, porter son nom de baptème, faire maigre le vendredi, réciter le Pater et l'Ave Maria. Pas un mot sur les croyances, sur la foi, sur les vertus, ni même sur les sacrements. Ainsi, malgré les oppositions évidentes entre christianisme et islam, pour ces chrétiens de base que sont nos renégats, la religion est seulement un ensemble de paroles, de gestes, de comportements, de ce côté de la Méditerranée aussi bien que de l'autre. Ainsi, paradoxalement mais de façon bien compréhensible, se trouvent unies dans une même conception religieuse les deux grandes croyances ennemies.

Les réalités chrétiennes, plus intérieures, seraient-elles donc absentes de ces procès? Evidemment non. Nous avons vu ci-dessus à plusieurs reprises, et même dans tous les cas, surgir ces affirmations du renégat: "je suis resté chrétien de coeur”, “je n'ai jamais cru que la secte des Turcs pouvait sauver 
les gens”, “je n'ai jamais cru ni voulu croire à leur secte”, “je n'ai jamais abandonné la foi du Christ, au contraire j'ai toujours eu l'intention de retourner à la dite sainte foi du Christ", etc. Cela paraît assez clair, même si passablement suspect, vu les circonstances et l'enjeu. Mais voyons les documents. Voici d'abord les trois réconciliations opérées par l'official de Marseille. Ici, nous n'avons pas le procès-verbal de l'interrogatoire mais seulement l'acte rédigé par le greffier et résumant la procédure. Le renégat, dans ces document, ne parle pas à la première personne. Par ailleurs la formule qui nous intéresse est chaque fois reproduite pratiquement à l'identique. Sans doute la réponse à une question précise et fermée, toujours la même et rédigée en latin - alors que dans les procès italiens si la question est en latin la réponse est en italien - comme celle de Renaud Cappus: "Il persévéra toujours intérieurement, fermement et assidûment, dans la religion chrétienne". Voici donc un stéréotype, que l'on peut considérer comme soufflé par l'official, et que nous ne pouvons donc retenir comme expression spontanée du renégat ni en déduire une quelconque vision religieuse.

Du moins les réponses des cinq renégats aux tribunaux italiens sont-elles plus fiables? Considérons les circonstances précises dans lesquelles le renégat parle de sa religion en termes d'intériorité. Inutile de tergiverser: chaque fois, c'est en réponse à une question précise du tribunal. Pour éviter une multiplication fastidieuse, prenons un seul exemple, celui de Jean Fabre. Il a d'abord fait un long récit de vie, au cours duquel il ne parle de religion qu'en termes de pratiques extérieures. Alors l'inquisiteur lui demande

$\mathrm{Q}$ - "si du temps où il resta avec le dit Amat Baïs il ne fit jamais acte de la secte musulmane ou d'adhérer à elle par esprit ou en intention,

$\mathrm{R}$ - Je n'ai jamais fait aucun acte de Turc ni même en pensée, au contraire je me suis toujours recommandé à Dieu bénit pour qu'il m'aide à sortir de leurs mains, ni même je n'ai jamais cru ni pensé croire à leur secte.”

La régularité avec laquelle s'observe d'une part l'absence de considérations chez les renégats sur leur adhésion intérieure au christianisme et, d'autre part au contraire, leur présence suivant toujours une question très fermée qui, comme on a pu le constater avec l'exemple proposé, dicte presque la réponse, conduit à une double réflexion. La première est que, spontanément, les renégats ont seulement une approche très concrète de leur christianisme, faisant l'impasse sur leur conscience intime. Quoique nous ne puissions généraliser à partir d'eux, puisqu'ils représentent des sortes de cas “aberrants" ou tout au moins exceptionnels en terre chrétienne, on peut toutefois se 
demander si ce n'était pas la démarche commune de leurs concitoyens et correligionnaires.

La seconde réflexion est que les clercs ne se reconnaissent pas dans cette vision du christianisme: ils ne la partagent évidemment pas. Pour eux la religion du Christ est avant tout une croyance où l'intention joue un rôle capital, à vrai dire le premier. Nous le vérifierons ci-dessous avec les indications que donnait le Saint-Office aux evêques sur les modes d'interrogatoires. Sur les huit questions indiquées, trois se modulent expressément sur ce registre (les $\mathrm{n}^{\circ} 5$, 6 et 7 du schéma proposé plus bas). Les cinq procès confirment: le tribunal interroge toujours le renégat sur cet aspect, alors que celui-ci ne l'aborde jamais de lui-même. Sur cette question du moins, malgré une vision commune de la religion qui la considérait comme un fait culturel concret immergé dans une civilisation matérielle, se lit la divergence des regards clérical et laïc sur le christianisme qu'ils partageaient: pour le premier, il est d'abord une affaire d'adhésion personnelle que l'on peut appeler la foi; le second le saisit comme un mode de vie et, dans le meilleur des cas, comme une morale. Ainsi la grande cause de l'intériorisation du christianisme, oeuvre entreprise par les clers depuis des siècles déjà, était loin encore d'être gagnée.

Belle trouvaille, ironiseront certains: il n'y a là rien de neuf. Voire! En effet, si d'autres études portant sur la religion courante en terre traditionellement chrétienne confirmait cette conception selon laquelle le christianisme est seulement, principalement ou d'abord, un mode de vie et non une adhésion personnelle pour la mojorité de la population théoriquement évangélisée et cléricalement encadrée, il conviendrait de revoir nos idées sur le fonctionnement religieux de cette ancienne société. Tomberait alors cette sorte de malentendu qui consiste pour l'historien à considérer comme "chrétiens" des gens qui vivent seulement et apparemment comme tels; s'amorceraient peutêtre de nouvelles recherches accompagnées de nouvelles questions et de nouveaux modes d'enquête; surgirait l'ancienne et toujours rénovée question inévitable de la nature du christianisme vécu.

Outre ces interrogations majeures, sur lesquelles débouche l'analyse du contenu des informations concrètes, tout aussi intéressant semble le "cas" offert aux canonistes par ces renégats de retour en Chrétienté: ils représentent un "modèle" juridique d'où découle une procédure propre; la variété des situations cependant explique la différence de traitement qui leur est appliqué avant de les réinsérer dans la communauté chrétienne. 


\section{L'embarras juridique}

Du point de vue du droit ecclésiastique, le droit canon, les renégats posaient un véritable problème. Il est clair que ce sont des apostats. Il est tout aussi évident que l'apostasie relève de l'Inquisition. Le Dictionnaire des inquisiteurs (Valence, 1494) déclare: "L'apostat, en abandonnant sa foi, pèche bien plus gravement que l'infidèle demeurant dans sa perfidie... L'apostasie est la plus infâme des infâmies, l'infâmie la plus détestable qui soit". ${ }^{11} \mathrm{Et}$ parmi les trois types d'apostasie que distingue le manuel des inquisiteurs de $\mathrm{N}$. Eymerich et $\mathrm{F}$. Pena, publié au seizième siècle, la troisième est celle du "chrétien qui nie une vérité de foi". Malgré la longueur du passage, lisons ce qu'il prévoit pour l'apostat:

Il va de soi que ce type d'apostasie retranche totalement de l'Eglise et de la foi catholique celui qu'il concerne. On traitera cet apostat en hérétique et en infidèle, et comme tel il sera processé. Impénitent, il sera livré au bras séculier. Repenti, il abjurera et sera traité en hérétique pénitent. Ceux qui ont apostasié par peur de la mort, mais qui sont restés fidèles dans leur coeur, ne sont pas à proprement parler des hérétiques. Mais l'Eglise doit juger du for extérieur, elle doit donc en conséquence les considérer comme tels. Comme tels ils seront jugés et, s'ils abjurent, ils seront pardonnés et condamnés à la réclusion perpétuelle, comme des hérétiques pénitents. N'ont-ils pas craint la mort davantage que l'inimitié constante de la foi du Christ? Ne vaut-il pas mieux mourir qu'apostasier? ${ }^{12}$

Mais l'Inquisition romaine, créée en 1542, pouvait-elle raisonnablement traiter ainsi, dans les années 1590 , les milliers de renégats qui allaient affluer et se retrouver devant ses tribunaux? Le prisons n'y auraient pas suffi. Par ailleurs la position du manuel est discutable. Le chrétien, comme tout homme, n'a-t-il pas le devoir premier et prioritaire de sauver sa vie? Si le martyre est une vocation spéciale de Dieu, il ne saurait constituer un devoir. De plus, en l'occurrence, les clercs se trouvaient pris à leur propre piège. Si le christianisme est supérieur à l'islam, mieux, s'il représente la seule voie de salut pour l'au-delà, comment refuser l'abjuration à ceux qui ont encouru mille dangers pour revenir en Chrétienté? D'autant que l'application de peines trop sévères aux réfugiés aurait vite été colportée en terre barbaresque et aurait pu décourager bien des anciens chrétiens à rentrer. Ainsi les tribunaux ecclésiastiques tendirent vers l'indulgence, sans tomber toutefois dans le laxisme. En effet sous l'aspect religieux, déjà relativement complexe, se trouvait également un aspect politique. Si les renégats repentis pouvaient 
fournir des renseignements utiles aux puissances chrétiennes sur les armements et dispositions militaires et maritimes des Barbaresques, on ne pouvait exclure a priori qu'en leur sein ne se cache quelque espion venu de l'est. Enfin se posait une difficulté de procédure: de quelle instance les renégats relevaient-ils? De l'officialité diocésaine ou de l'Inquisition romaine? Pour toutes ces raisons, les renégats troublaient la justice ecclésiastique qui ne savait trop comment procéder.

Nous lisons cet embarras dans les échanges de correspondance auxquels se livrèrent les tribunaux épiscopaux d'Italie et le Saint-Office de Rome à propos de trois de nos renégats. L'archevêque de Santa Severina, Alfonso Pisano ${ }^{13}$, écrivait au cardinal romain le 15 décembre 1593. Il avait interrogé Vital Redon sur son apostasie et il joignait à sa lettre le procès-verbal de l'interrogatoire, car le comte de Santa Severina soupçonnait le prisonnier d'être un espion des Turcs ("per dubbio che non sia spia di Turchi"). Pourtant cela lui semble peu vraisemblable car le renégat est un étranger qui ne parle pas bien la langue du pays et, de plus, il a l'oreille coupée. Aussi la charge d'espionnage fut-elle abandonnée.

Quoique apparement plus banal, le cas de Jacques Gérard embarrassait l'archevêque de Cosenza, ${ }^{14}$ en Calabre. Il écrivait le 21 mars 1592 au Saint-Office une lettre qui y fut enregistrée le 2 avril: "Dans les prisons de cette cour se trouve un jeune français de 28 ans qui fut pris enfant par les Turcs qui le circoncirent et lui firent renier la foi par force; il réussit à s'enfuir et a abouti ici où il m'a fait instance pour être réconcilié. C'est pourquoi j'ai jugé nécessaire d'écrire à votre S.I. pour qu'elle me commande ce que je devrai faire en tel cas...". ${ }^{15}$ La réponse du cardinal, datée du 22 avril, indique la marche à suivre:

Que votre S. examine avec diligence le dit Jacques, lui faisant raconter à plein le fait de sa capture, les modes, paroles et cérémonies qui se firent lorsqu'ils le firent renier et circoncire, quelles sortes de violence intervinrent; combien de temps il est resté ensuite chez les Turcs; s'il s'habillait à la turque et a observé leurs faux rites et finalement l'interrogera s'il ne s'est jamais détaché, de quelque façon, en esprit, de la sainte foi catholique et a adhéré à la secte impie de Mahomet, ou bien s'il a cru que quelqu'un puisse se sauver hors du giron de la sainte Eglise catholique. Et, s'il répond bien et catholiquement, que V.S. le condamne à abjurer secrètement devant ce tribunal comme véhémentement suspect d'apostasie de la sainte foi catholique à la secte des Turcs et l'absolve de toutes les censures et peines ecclésiastiques lui imposant quelques pénitences salutaires laissées à l'arbitraire de V.S. Mais s'il a confessé avoir eu 
quelque opinion erronée et s'être détaché, en tout ou partie, de la sainte foi catholique en quelque façon avec son esprit à la dite secte impie, elle le fera abjurer comme apostat formel, l'absolvant et lui imposant les pénitences comme dessus, lesquelles pourtant, dans ce cas, devront être plus graves et, si besoin, elle le fera instruire par une personne intelligente, catholique et zélée avec charité... Elle l'interrogera encore particulièrement si, en ces régions, il eut connaissance de quelque autre chrétien renégat, lui faisant spécifier le nom et prénom qu'il avait lorsqu'il était chrétien, le nom de sa patrie et nation, puis elle enverra copie de l'ensemble à cette sacrée congrégation. ${ }^{16}$

C'est dans cette lettre que le Saint-Office donne les indications les plus précises et les plus complètes sans doute parce que l'évêque était nouveau, nommé l'année précédente, et que c'était peut-être le premier procès qu'il avait à instruire à l'encontre d'un renégat. Le canevas d'interrogatoire recommandé porte ainsi sur huit points:

1. Récit de la capture;

2. Modes, paroles et cérémonies du reniement et de la circoncision ainsi que les violences subies;

3. Durée du séjour chez les Turcs;

4. S'il a observé leurs faux rites;

5. S'il s'est éloigné de la sainte foi catholique;

6. S'il a adhéré à la secte impie de Mahomet;

7. S'il a cru qu'on pouvait être sauvé hors de l'Eglise;

8. S'il a connu d'autres renégats: leurs nom, prénom, nation.

Tels sont donc les points cruciaux sur lesquels il convient, selon le Saint-Office, d'interroger afin de se faire une opinion, juger en connaissance de cause, apprécier le cas et y adapter la sentence. Nous pourrions soumettre nos huit cas à cette grille de lecture: analyser chaque paramètre et vérifier pour chacun de nos renégats comment les diverses variables peuvent se combiner. Remarquons au passage combien l'intenton est un élément capital aux yeux du clergé: le christianisme ne peut se contenter d'une adhésion et d'une pratique simplement extérieures.

La lettre du cardinal de Santa Severina arriva le 12 mai à Cosenza, mais le détenu n'y était plus. Il avait été envoyé avec la "chaîne" des prisonniers dans les prisons de Naples. L'évêque avait ainsi résolu le problème, du moins en ce qui le concernait.

De la même veine devait être la lettre adressée à l'institution romaine par l'évêque de Mileto, ${ }^{17}$ en Calabre, sur le cas d'Augustin Borré. Nous ne 
disposons pas de celle-ci mais de la réponse datée de Rome le 26 novembre 1594. Le cardinal répond qu'il convient de procéder selon la forme du pontifical. Il faut interroger le renégat particulièrement

s'il a jamais cru que la secte des Turcs fût bonne et qu'en elle on pût faire son salut, s'il a adhéré à celle-ci, doutant de la sainte foi du Christ et, suivant ce qu'il répondra, V.S. le recevra comme comparaissant spontanément et lui fera abjurer cette apostasie ou comme apostat formel, ou bien comme véhémentement ou légèrement suspect d'apostasie, suivant la qualité des confessions et les circonstances du fait et de la personne. Puis elle pourra l'absoudre comme ci-dessus ou comme apostat simplement a cautela comme suspect d'apostasie en lui imposant quelques pénitences salutaires selon le droit et la coutume. Elle fera faire du tout acte public et pourra m'en envoyer copie. Cela V.S. peut le faire juridiquement de par son autorité comme pour un hérétique encore qu'il y ait la réserve dans la bulle In Cena Domini; mais, pour le for interne, qu'elle se reporte à la dite bulle. Pour le reste, V.S. fera oeuvre de grande charité de le recommander chaleureusement à l'ill. S. duc de Monteleone qui, comme elle le lui a écrit, l'a pris chez lui pour qu'il soit instruit dans la sainte foi et acheminé sur la route de la piété chrétienne comme il convient... 18

Le tracas des évêques est par lui-même déjà révélateur: la conduite à tenir n'est pas encore tracée. Ils ne savent comment traiter ces déviants qui ne sont pas vraiment des hérétiques. Certes ils sont apostats, mais ont été forcés de renier leur foi. En revanche les deux réponses du Saint-Office, faites à deux évêques différents et à plus de deux ans l'une de l'autre, montrent que la règle est en train de se mettre en place. Rappelons que si l'Inquisition romaine fut créée 1542, elle ne fut jamais officiellement reçue dans le royaume de Naples, lequel se trouvait également exempt de l'Inquisition espagnole. Pourtant le Saint-Office de Rome y était présent de fait, pouvant y nommer des substituts et le vicaire général du diocèse s'intitulait également commissaire du SaintOffice. On voit ici la singularité du fonctionnement de l'inquisition en Italie du Sud et la souplesse de ses institutions. ${ }^{19}$

Pour la réconciliation des apostats, comme des hérétiques et autres déviants de la foi, la référence obligée reste, en cette fin du seizième siècle, la bulle de Paul III, dite In Coena Domini parce que donnée le jeudi saint 1536. Le cardinal du Saint-Office ne manque d'ailleurs pas d'y renvoyer l'évêque de Mileto. Datant d'avant la création de l'Inquisition romaine, le document pontifical précise la procédure que les tribunaux épiscopaux doivent suivre dans les cas d'hérésie et d'apostasie. ${ }^{20}$ 
A strictement parler, ces cas relevaient de l'autorité épisopale. Le droit canon reconnaît expressément à l'officialité le droit de recevoir les abjurations que l'autorité exige par prudence ("ad cautelam") dès lors qu'il y a présomption de culpabilité au for externe, même si la conscience du converti (le for interne) atteste sa bonne foi. Pourtant les crimes d'hérésie relevaient de l'Inquisition. Et, depuis la création du Saint-Office à Rome en 1542, les conflits de juridictions ne sont pas théoriques. Le fait que les évêques du royaume de Naples prennent soin de prendre conseil auprès du Saint-Office à Rome en dit long sur l'influence de ce dernier. Certes le cardinal de Santa Severina rappelle à l'évêque qu'il a le pouvoir de juger le renégat, mais il tient à lui indiquer les distinctions majeures des divers cas possibles et à lui réclamer copie de l'ensemble de la procédure. Conseil et contrôle allaient de pair.

Il est parfaitement clair que toutes les interrogations doivent permettre de déterminer la gravité de l'apostasie et la sincérité du retour en chrétienté. Ainsi, schématiquement et théoriquement, se trouvent opposés deux types extrêmes. Le premier serait le cas du "martyr": enlevé jeune, ayant subi longtemps de graves violences, finalement contraint à renier mais resté fidèle à sa foi chrétienne, ayant saisi la première occasion pour s'enfuir et se présentant spontanément devant le tribunal ecclésiastique. C'est le renégat "idéal", correspondant au modèle que les rédemptoristes ne cessèrent de colporter au dix-septième siècle dans leurs tournées de sensibilisation en occident afin de réunir les sommes nécessaires au rachat des captifs. Le contre-type serait le jeune homme enlevé de force également, ayant peu ou pas souffert durant sa détention si toutefois elle eut lieu, ayant rapidement renié le christianisme, vécu en "Turc" et pratiqué les rites musulmans, ayant cru sincèrement qu'il pourrait ainsi être sauvé, peu pressé de rentrer en chrétienté, voire contraint de le faire, et de se présenter à la justice, éventuellement soupçonné d'être un espion. Si les cas limites sont limpides, ils sont à ce point schématiques qu'ils restent quelque peu théoriques. Concrètement, nos renégats comme tous les autres, à quelques exceptions près, se situent entre ces extrémités. Les juges ont précisément pour tâche de situer chaque cas, d'apprécier la sincéritié des aveux ainsi que le caractère forcé ou non de l'adhésion à l'islam afin d'évaluer la responsabilité de chacun et déterminer en conséquence l'attitude à adopter et la sentence à donner.

L'affaire n'était pas simple, même une fois écartée l'accusation, toujours possible, d'espoinnage et admise l'apostasie. En effet refuser l'absolution était presque impossible: cela aurait ralenti le flot des retours en chrétienté. Mais l'accorder trop facilement, au point de transformer le jugement de réconciliation en pure formalité était tout aussi inacceptable: c'était galvauder 
la foi chrétienne. Par ailleurs les autorités cléricales constituant le tribunal étaient prisonnières de leurs conceptions religieuses. Persuadés de l'excellence incontestable du christianisme, ces clercs ne pouvaient imaginer un seul instant qu'un chrétien puisse librement renoncer à sa foi, c'est-à-dire à son salut éternel, pour adopter l'islam, c'est-à-dire la damnation. Aussi, malgré toutes les précautions prises, le tribunal était-il enclin à accorder crédit au récit du renégat. L'aspect quelque peu stéréotypé de celui-ci éveillait bien certains soupçons. Mais les renégats, d'une part, savaient quel discours les inquisiteurs attendaient et, d'autre part, le tribunal se trouvait passablement piégé face au jugement à émettre.

Ainsi juges et accusés, plus ou moins sciemment, acceptaient les fondements d'une même conception religieuse, produisaient ou recevaient le même discours, se conformaient au rôle respectif que chacun attendait de l'autre suivant le modèle de répartition sociale et culturelle admis. Tout l'art du clerc consistait donc à interroger pour connaître une certaine vérité acceptable, sans pour autant rechercher à tout prix la vérité qu'il n'était pas prêt à entendre et sans faire preuve toutefois d'une indulgence qui aurait paru complice, donc coupable. De son côté, le récit du renégat devait paraître crédible, notamment en ce qui concernait sa résistance à demeurer chrétien secrètement. A défaut d'être véridique, sa version devait pour le moins sembler plausible et vraisemblable. A aucun moment le juge ne pouvait laisser paraître au renégat qu'il ne croyait pas son récit s'il l'absolvait tout de même. Loin de vouloir le piéger, dans cette circonstance particulière, le clerc attendait la plus petite possibilité pour conclure à la sincérité et à la fidélité du malheureux martyr chrétien persécuté par les Turcs. Ainsi le jeu était relativement subtil pour le juge autant que serré pour l'accusé, du moins si nous admettons, ce que nous pouvons éventuellement savoir par ailleurs, que certains d'entre eux se convertirent volontairement à l'islam ou que, du moins, forcés à renier, ils devinrent ensuite de bons musulmans.

Le tribunal marseillais ne se posa pas tant de questions. L'évêque de la cité phocéene se trouvait moins embarrassé que ses confrères italiens, tiraillés entre le Saint-Office et l'officialité. D'abord parce que, n'en doutons pas, les cas de ces retours de Barbarie ne devaient guère être rares dans ce port méditerranéen. L'enquête mériterait d'être menée. Un rapide sondage dans le fonds de l'Amirauté de Marseille, quelques registres notariés de cette ville et dans les papiers Grandchamp sur Tunis a révélé 116 Provençaux vivant en Barbarie entre 1565 et 1600 dont 72 Marseillais, 34 esclaves et 36 renégats. $^{21}$ Les actes notariés ne manqueraient pas de révéler d'autres individus et d'autres aspects. Deux exemples pourront convaincre. Voici d'abord un acte 
du 17 octobre 1562: il s'agit d'une “commission”, donnée par un Marseillais à un marin de la ville accompagnée de 50 écus, pous aller racheter Jaumet Mortier de Marseille

debtenu prisonnier entre les mains des infidelles éz parties d'Argier ainsi qu'ils ont est advertis par une lettre du dit Jaumet Mortier escripte au dit Argier le second du présent moys estant par laquelle faict mention de bailher commission à patron Carolin Deydier du dit Marseille de le rachepter et de luy bailher argent pour ce fayre.

L'affaire a été conclue et le prisonnier rendu puisque l'acte, cancellé le 2 avril 1565 , précise alors que chaque partie s'estime satsifaite de l'autre. Jaumet avait-il renieé ou, au contraire, résisté? Nous ne le savons pas. ${ }^{22}$

En revanche l'autre cas est des plus explicites. Le 26 janvier 1555, le seigneur de Venelles et de Mimet, deux villages près d'Aix-en-Provence, Esprit Etienne Chaussegros, dicta son testament dans lequel nous pouvons lire:

Plus, sachant le dit testateur avoir ung filz nommé Jehan Estienne Chaussegros, lequel a délaissé la religion chrestienne et a prins et s'est mis à la loy maiométant et c'est randu Turc à la cité d'Argiers et consumé mariage par coppulation charnelle avec une Turque,... à ceste cause et aultre... le mourant l'a exhérédé et exhérédé de tout son dit bien...

Et de lui léguer tout de même 100 florins pour qu'il ne puisse rien réclamer d'autre. Voilà qui nous permet de saisir ces renégats qui, au contraire de Jaumet Mortier rêvant de revenir dans leur patrie, semblaient se satisfaire de leur sort et n'envisageaient pas de retour, du moins pas encore. ${ }^{23}$

Par ailleurs si l'official de Marseille ne manifesta aucun état d'âme pour recevoir les abjurations et réintégrer les renégats dans la communion des chrétiens, c'est aussi parce que l'Inquisition romaine n'était pas reçue en France, et ne le fut jamais. En conséquence l'hérésie, comme l'apostasie, relevait du tribnal épiscopal. Nous pouvons vérifier la différence d'attitude entre les autorités ecclésiastiques locales d'Italie et celles de France avec le cas de Honoré Dalhot, déjà rencontré plus haut. Ce jeune Marseillais a moins de seize ans lorsque, de retour dans sa ville, il se trouve devant l'official, le 8 septembre 1591. Il avait été embarqué sur une trirème turque pour la Corse, avait réussi à s'enfuir puis à monter sur une trirème florentine "avec laquelle, ces jours derniers il arriva à Marseille." Le cas est donc classique et parfaitement simple, encore que le jeune homme ait renié sa foi, soit circoncis et ait 
vécu à la turque. L'official le réconcilie sans difficulté et sans pénitence en présence de Luc et Pierre Dalhot, ses parents certainement. ${ }^{24}$

Cependant, ce que ne dit pas notre Marseillais, et que nous savons par une toute autre source, c'est la tentative qu'il avait déjà faite à Florence. En effet la même année 1591 l'archevêque de Florence, le nonce aussi bien que l'inquisiteur, auprès desquels il avait demandé à abjurer prétextant de l'incompétence de leur instance judiciaire, lui avaient refusé la réconciliation et le contraignirent à faire appel à Rome. Le mémoire explicatif est joint à la lettre du cardinal de Sainte Séverine, qui répond à l'inquisiteur que les cardinaux inquisiteurs généraux ont résolu le cas d'Honoré et lui demandent de recevoir sa confession et son abjuration. Mais, à cette date, l'affaire était déjà réglée puisque, comme nous l'avons vu, dès le 8 septembre, Honoré Dalhot avait rejoint Marseille et abjurait entre les mains de son official, alors que la lettre cardinalice est datée du 27 septembre 1591. Il est cependant utile de noter à cette occasion qu'à Florence ni l'archevêque, ni le nonce, ni l'inquisiteur n'ont cru pouvoir faire abjurer le jeune renégat, le renvoyant au Saint-Office de Rome. Au contraire, l'évêque de Marseille ne fit aucune difficulté. 25

Sur nos huit renégats, nous connaissons la sentence pour cinq: ils sont absous avec une pénitence légère ou même, dans deux cas, sans pénitence du tout. Pour deux autres, Jacques Gérard et Vital Redon, l'issue ne fait guère de doute, dès lors surtout que l'accusation d'espionnage fut abandonnée contre ce dernier: ils durent certainement recevoir également l'absolution. Sept des huit renégats présentèrent donc suffisamment de garanties de sincérité pour être réintégrés dans le giron de l'Eglise romaine. Tout au moins il n'a pas été jugé nécessaire au tribunal de pousser plus avant ses investigations. On constate ainsi que, à titre d'indice, la durée du séjour en Barbarie n'a pas grande importance, variant ici entre six mois et 14 ans. De même ce qui importe, aux yeux des clercs, n'est pas tant le reniement et la circoncision que la durée séparant ces rites de la capture en mer et la vie menée par le renégat au sein de la population barbaresque. Ainsi le moins coupable est Jean Fabre: il n'est resté que six mois en Barbarie où il était prisonnier, n'a ni renié ni été circoncis, n'a jamais accompli aucun rite musulman et s'est enfui à la première occasion; il est vrai qu'il était sensiblement plus vieux que la moyenne puisqu'il avait 26 ans. Mais les six autres, on l'a vu, furent également reçus à pénitence. Reste le dernier, le huitième, qui se rapproche le plus du contre-type.

Le malheureux Léon de Olivier ne paraît guère sincère aux yeux de l'inquisiteur romain qui ne se fie pas à ses déclarations. En effet le Marseillais 
a renié et a été circoncis seulement un mois après sa prise, s'est habillé et a vécu en musulman, était libre en Barbarie, rameur volontaire sur les galères turques puis même soldat pendant trois ans. Comment aurait-il pu, dans ces conditions, maintenir sa foi chrétienne, ce qu'il affirme cependant? D'ailleurs son retour n'a pas été volontaire et ne constitue pas une évasion puisqu'il a été fait prisonnier en mer par un bâtiment chrétien. Enfin, dernière circonstance accablante pour qui prétend être resté un fidèle du Christ même secrètement, il se révèle incapable de reciter Credo et Salve Regina. Rien d'étonnant à ce qu'il soit livré à la torture, le seul des huit d'ailleurs. Malgré l'absence de la sentence, nous pouvons raisonnablement conjecturer que, même s'il fut finalement absous, il se retrouva prisonnier sur les galères chrétiennes. Il paraît assuré que les soupçons étaient fondés et que, même s'il fut converti de force, le jeune Marseillais était devenu un bon et vrai musulman et que seul le sort malheureux le ramena en Italie. Ainsi, contrairement à l'image traditionnelle véhiculée en chrétienté, peut-être pour Léon de Olivier et sûrement pour Jean Etienne Chaussegros - mais pour combien d'autres? -, la liberté souhaitée pouvait avoir le parfum de la Barbarie et le salut espéré la couleur de l'islam.

\section{Conclusion}

L'échantillon analysé ici et constitué par seulement huit renégats marseillais, malgré son étroitesse, a permis d'abord de vérifier les conclusions auxquelles les études antérieures étaient parvenues, que ce soit pour l'âge et la profession, les circonstances de la capture en mer, le reniement, les lieux et les conditions du séjour en Barbarie, les motivations officielles du retour, bref la trame générale du "récit de vie".

Il a également permis une approche du type de religion que ces hommes, au-delà de ce qu'ils avaient effectivement vécu, pensaient vivre. Le fait religieux était à leurs yeux une composante, parmi d'autres, quoique probablement de première importance, d'un mode d'être, d'une civilisation, d'une culture. Il n'est pas outré de penser qu'ils ne pouvaient imaginer un chrétien s'habiller à la turque et pas davantage un musulman faisant maigre le vendredi. Pour eux non seulement telle abstention, telle parole, tel geste est signe d'appartenance religieuse, mais il est signe d'identité et cette identité est tout à la fois ethnique, culturelle et religieuse. A ce niveau, juges et renégats partagent la même anthropologie religieuse.

Cette façon d'appréhender la vie spirituelle dans la réalité quotidienne ne regarde pas seulement, contrairement à ce que l'on pouvait penser, l'islam. 
Le christianisme est également concerné. Son originalité n'apparaît pas dans l'affirmation des grands dogmes fondamentaux, tels que la Trinité, l'Incarnation, l'Eucharistie, même exprimés de façon maladroite, schématique, voire erronée. Ces grandes vérités de foi ne sortent jamais, sous quelque forme que ce soit, de la bouche de nos renégats qui appartiennent tous, pour autant qu'on le sache, aux couches populaires de la société marseillaise. Il n'y a rien d'étonnant à constater que la population appréhende sa religion à partir non pas de concepts qui pour être fondamentaux n'en sont pas moins abstraits mais plutôt des éléments concrets de la religiosité, voire de coutumes culturelles.

Il est plus surprenant de voir nos Marseillais impliquer si peu leur âme dans la perspective du salut éternel. Il est possible que les renégats en général, et nos hommes en particulier, ne soient pas représentatifs de la population chrétienne de leur temps. Il est vraisemblable que leur aventure ait pu les rendre parfois de vrais musulmans et souvent quelque peu sceptiques. Il reste remarquable de voir ainsi absentes chez eux la conviction personnelle, l'adhésion de la conscience, l'intériorisation de la croyance, à l'inverse des clercs qui les interrogent pour lesquels, au contraire, là est le point essentiel, là est le coeur du christianisme. Le face à face judiciaire révèle la distance établie entre les uns et les autres que ne sépare pas seulement une table: deux conceptions culturelles se rencontrent ou mieux se croisent.

Ces documents enfin permettent de vérifier que c'est tard dans le seizième siècle, et même au début du dix-septième, que la procédure propre aux renégats se met en place, après des décennies de tâtonnements. Non pas qu'il y ait eu hésitation sur la nature du crime, sur le fond. C'est plutôt de la complexité judiciaire créée en Italie par l'institution de l'Inquisition romaine, fondée en 1542, que sont venus embarras et hésitations. De fait nous pouvons constater que le même crime d'apostasie pouvait, selon les lieux, relever de trois instances différentes; l'Inquisition espagnole dans les domaines du Roi Catholique, dont, par exemple, le royaume de Naples était exempt; en France, comme dans tous les pays où le Saint-Office romain n'avait pas été reçu, les officialités diocésaines, comme par le passé; en Italie enfin, à l'exception évidemment des Etats pontificaux, la concurrence jouait entre les tribunaux épiscopaux et l'Inquisition romaine. Celle-ci toutefois, refusant d'affronter directement les évêques, jouant en finesse, finit par établir plutôt, grâce à d'habiles attitudes, une collaboration avec les diocèses, comme nous l'avons vu avec ceux de Mileto, Cosenza ou Santa Severina. Les cardinaux inquisiteurs de Rome répandirent ainsi, peu à peu, un modèle d'interrogatoire, 
uniformisant progressivement la procédure, tout en feignant de respecter l'autorité épiscopale dans la péninsule.

Ainsi les "morceaux de vie" de ces quelques Marseillais, embarqués malgré eux dans une aventre qu'ils n'avaient sans doute même pas imaginée, nous apprend autant sur eux-mêmes que sur ceux qui les interrogeaient, sur la conception religieuse des uns et des autres, sur le cas juridique qu'ils offraient lorsque, plus ou moins volontairement, ils revenaient en Chrétienté. On voudrait suivre ces trajectoires humaines après la réintégration dans l'Eglise: quelles leçons tirèrent-ils de leur détour barbaresque? On aimerait savoir quelle vie religieuse ils menèrent ensuite: à quelles conclusions personnelles aboutirent-ils, suite à leur expérience religieuse double et comparative?

Une piste mérite une attention spéciale. En 1601, Hélène Dupuy, fille de Georges et habitant Marseille, était l'épouse d'André Etienne Chaussegros, ce dernier étant le frère de celui qui s'était converti à l'islam et que nous avons vu déshérité par son père. Or un Jean Dupuy, autre Marseillais, était reçu habitant de Genève en décembre 1572. Les rénégats d'Alger et les protestants de Genève verraient-ils leur route se croiser à Marseille? Les diverses voies du non conformisme religieux se rejoindraient-elles parfois? Voilà qui ouvrirait des perspectives insoupçonnées pour l'étude et la compréhension de la dissidence et des transfuges au seizième siècle. ${ }^{26}$

\section{Université de Provence}

\section{Notes}

1. B. Bennassar, "Conversion ou reniement? Modalités d'une adhésion des chrétiens à l'Islam (XVI'e-XVII' siècles)". Annales E.S.C., 1988, no. 6 (nov.-déc), p. 1349-1366. Voir surtout B. et L. Bennassar, Les chrétiens d'Allah. L'histoire extraordinaire des renégats, $X V I^{e}-X V I I^{e}$ siècles (Paris, Perrin, 1989).

2. A la page 167 , le nombre indiqué est " 28 ", mais c'est une erreur car en comptant les Marseillais indiqués en annexe (p. 477-480) on arrive bien à 29. Je tiens ici à remercier B. Bennassar qui, apprenant que je m'intéressais à la question, m'a gentiment adressé les fiches qu'il avait établies pour les 23 renégats marseillais qu'il avait alors repérés, et cela dès avril 1987.

3. Paris, B.N. Ms Lat. 8994. Registre indiqué dans: John Tedeschi, "The dispersed Archives of the Roman Inquisition", in G. Henningsen and J. Tedeschi, The Inquisition in Early Modern Europe. Studies on Sources and Methods. (DeKalb: Northern Illinois University Press, 1986), p. 28, note 30. Et Archives départementales, Bouches-duRhône, 381 E. 390, actes aimablement indiqués par Wolfgang Kaiser, à qui je tiens à exprimer ma gratitude.

4. Paris, B. N. Ms Lat. 8994, fo 252 (Jacques Gérard); fo 268 (Jean Fabre), fo 284 (Léon de Olivier); fo 302 (Vital Redon); fo 362 (Augustin Borré). 
5. Le seul diocèse provençal intitulé "Saint-Paul" est celui de Saint-Paul-Trois-Châteaux. Né en 1575, notre Provençal dut être confirmé vraisemblablement par Antoine Gaume, évêque de ce diocèse de 1585 à 1598.

6. Ce prénom serait réservé aux rénégats, du moins nous ne le connaissons pas porté par des musulmans de naissance: voir de B. et L. Bennassar, op.cit, p. 332.

7. Archives départementales, Bouches-du-Rhône, 381 E 390, fo 162,163 et 165 respectivement. Les actes sont rédigés en latin tandis que, dans le registre de l'Inquisition romaine, ils le sont en italien.

8. La formule du passage à l'islam, largement confirmée par des témoignages massifs, peut se traduire par: "Il n'y a de Dieu que Dieu et Mahomet est son prophète". On peut vérifier plus haut avec quelle fantaisie la mémoire de Jacques Gérard et celle de Léon de Olivier avaient retenu la formule et sa traduction.

9. L. Rostagno, Mi faccio Turco. (Roma, Istituto per l'Oriente C.A. Nallino, 1983), p. 60-72: "L'islam dei rinnegati"; B. et L. Bennassar, op.cit, p. 325-332: "L'habit fait le musulman".

10. Cette interrogation n'a pas complètement échappé à ces historiens, mais elle a davantage fait l'objet de quelques remarques éparses de leur part plus que d'un développement circonstancié.

11. Louis Sala-Molins, Le dictionnaire des inquisiteurs, Valence, 1494. (Paris, Galilée, 1981).

12. N. Eymerich \& F. Pena, Le manuel des inquisiteurs, par L. Sala-Molins. (Paris-La Haye, Mouton), p. 83-84.

13. Alfonso Pisani fut évêque de Santa-Severina de 1587 à 1623 . A ne pas confondre avec le cardinal de Santa-Severina, Jules-Antoine Santorio, juge de l'Inquisition à Rome et signataire de ces lettres adressées aux divers évêques dont il est ici question, neveu de Paul IV, vicaire général du cardinal Alfonso Caraffa, archevèque de Naples; archevèque de Santa-Severina en 1566, cardinal en 1570, mort en 1602.

14. Jean-Baptiste Constanzi, évêque de Cosenza de 1591 à 1617.

15. Paris, B.N. Lat. 8994, fo 253.

16. Ibid., fo $255-256$.

17. Antoine de Tufo, évêque de Mileto de 1585 à 1606.

18. Paris, B.N. Lat. 8994, fo $364-364 v^{\circ}$

19. Voir sur cet aspect les pages très claires de J.-M. Sallmann. Chercheurs de trésor et jeteuses de sort. (Paris, Aubier, 1986), p. 54-67.

20. La bulle dont il est question est celle de Paul III "Consueverunt Romani Pontifices" du 13 avril 1536. Elle est consacrée à divers types de délinquants dont les hérétiques: $\mathrm{C}$. Coquelines, Bullarum, privilegium ac diplomatum romanorum pontificum amplissima collectio. Rome, 1745, T. IV, pars 1, 1, 140-143. 
21. Archives départmentales, Bouches-du-Rhônes, IX B 171. Registre des actes et contrats passés à la chancellerie du consulat de France à Alger P. Grandchamp, La France et la Tunisie à la fin du XVIe siècle (1580-1600), Tunis, 1920; t. 1 .

22. Archives départementales, Bouches-du-Rhône, 390 E 117 fo $1264 v^{\circ}$

23. Signalé dans Arch. Com. Marseille, Fonds Bertas, 20 ii 182; Arch. départ, B-du-R., 352 E $150, f^{\circ} 87 v^{\circ}$

24. Ibid., 381 E 390 , fo 165.

25. L. Rostagno., p. 15-16: Archives Générales du Royaume, Bruxelles, Arch. Eccl. 19283 ter 2, c. 36-37. L'évêque de Marseille est alors Frédéric Ragueneau (1572-1603).

26. Cette information est due à l'amabilité de Wolfgang Kaiser (lettre du 10 juin 1987). 